\title{
Amenorréia e osteoporose em adolescentes atletas
}

\section{Amenorrhea and osteosporosis in adolescents athletes}

\author{
Graziela MANTOANELLI ${ }^{1}$ \\ Maria Sylvia de Souza VITALLE 2 \\ Olga Maria Silverio AMANCIO²
}

$\underline{\text { RES U M O }}$

A participação feminina no esporte foi crescendo ao longo dos anos. Trabalhos científicos têm mostrado os benefícios trazidos por esta prática para a saúde da mulher, mas quando se trata de esporte competitivo podem surgir problemas. A complicação mais comum descrita pela literatura é a "tríade da atleta" , a qual envolve três processos: o distúrbio alimentar, a amenorréia e a osteoporose. A amenorréia atinge, nos Estados Unidos, até $66 \%$ das atletas de competição, e algumas de suas possíveis causas são: perda de peso, excesso de treino, quantidade insuficiente de gordura corporal, perda de estoques específicos de gordura corporal e dieta inadequada. Como conseqüência da amenorréia, a esportista pode desenvolver osteoporose precoce. Não se sabe ao certo a porcentagem de atletas com osteopenia, mas há indícios de que a falta de estrógeno, a dieta inadequada e o consumo insuficiente de cálcio serem fatores que as predispõem a desenvolver a doença.

Termos de indexação: puberdade, amenorréia, osteoporose, exercício, atletas, adolescente, dieta.

\section{A B S T R A C T}

The female participation in sports has been increasing over the years. Scientific studies have shown the benefits this practice brings to women's health, but, when it comes to competitive sports, problems may arise. The most common complication described by the literature is the "Female Athlete Triad", which involves three processes: the eating disorder, the amenorrhea, and the osteoporosis. In the United States, the amenorrhea afflicts up to $66 \%$ of the competition athletes, and some of its possible causes of are: weight loss, excessive training, insufficient quantity of body fat, loss of specific stores of body fat, and inadequate diet. As a

\footnotetext{
1 Curso de Nutrição e Metabolismo na Infância e Adolescência, Departamento de Pediatria, Universidade Federal de São Paulo/EPM. Rua Botucatu, 703, Vila Clementino, 04023-062, São Paulo, SP, Brasil. Correspondência para/ Correspondenceto: M.S.S.VITALLE. E-mail: vitalle.dped@epm.br

${ }^{2}$ Curso de Nutrição e M etabolismo na Infância e Adolescência, Departamento de Pediatria, Universidade Federal de São Paulo.
} 
consequence of the amenorrhea, the sportsw oman may develop precocious osteoporosis. It is not known for sure the percentage of athletes with osteopenia, but there are indications that the lack of estrogen, the inadequate diet, and the insufficient consumption of calcium are factors predisposing them to develop the disease.

Index terms: puberty, amenorrhea, osteoporosis, exercise, athlete, adolescent, diet.

\section{N T R O D U Ç Ã O}

Historicamente não havia encorajamento da participação feminina em jogos esportivos, pois presumia-se que a mulher não estava apta fisiologicamente para participar de atividade física de alta intensidade e longa duração. 0 ciclo menstrual sempre foi ligado a superstições, como a mulher menstruada não poder lavar o cabelo ou tomar banho. E ainda era questionado se a "delicadeza" das mulheres não poderia ser prejudicada pelos músculos que ganhariam, caso participassem de qualquer esporte.

Somente a partir de 1900 permitiu-se a participação feminina nas Olimpíadas, e antes de 1960 só era permitido à mulher correr até 800 metros. A maratona feminina foi incorporada a partir de 1984, evidenciando-se a falta de incentivo ao esporte feminino (Timmerman, 1996; Wiggins $\&$ Wiggins, 1997).

Nos Estados Unidos, em 1972, criou-se a legislação chamada Título IX, a qual fez com que as escolas dessem oportunidades, incentivo financeiro e benefícios iguais para homens e mulheres nos esportes. Antes dessa legislação, 1 em 27 meninas participavam dos esportes escolares; atualmente, em média, 1 em 3 participam, indicando aumento de $600 \%$ ou um total de 1,9 milhões de atletas femininas (Benson et al., 1995).

Com o aumento da participação feminina no esporte, notaram-se os seus benefícios: efeitos fisiológicos, como a prevenção de câncer de mama e de osteoporose; efeitos psicológicos, como a aceitação melhor do corpo e maior auto-estima; e efeitos sociais, evitando a gravidez na adolescência e 0 envolvimento com drogas e ocasionando maior assiduidade na escola, permitindo às adolescentes terem maior probabilidade de terminar o segundo grau. No entanto, apesar de todos estes benefícios que 0 esporte pode trazer para as adolescentes, quando se trata de modalidade competitiva, sempre pode haver riscos de injúrias e fraturas ou problemas relacionados à saúde (Callahan, 2000).

As atletas de competição apresentam elevada incidência de irregularidades menstruais, como amenorréia secundária, ciclos irregulares e ciclos anovulatórios, independentemente do tipo de esporte praticado. Na maioria das vezes, as mais jovens e aquelas submetidas a treino mais intenso, como as maratonistas, apresentam maior prevalência de irregularidade menstrual do que as demais (Timmerman, 1996).

Os distúrbios menstruais são relacionados tanto às desordens alimentares quanto à osteoporose. São processos discretos os quais requerem atenção por parte dos profissionais, com eles envolvidos.

O conjunto desses problemas foi descrito pelo American College of Sports M edicine como a "tríade da atleta", em virtude da sua elevada incidência e do risco que representa para a saúde das atletas. Ela foi evidenciada originalmente nas esportistas cujo baixo peso as favorecia (ginástica olímpica) ou naquelas com intenção de aumentar seu rendimento (maratonistas) ou ainda nos esportes nos quais havia classificação por peso (Wiggins \& Wiggins, 1997). Porém, hoje sabe-se que ela pode ocorrer em qualquer modalidade esportiva.

A melhor maneira de se entender a tríade é estudar cada componente separadamente, mas, como em todo processo de doença, ela ocorre de maneira contínua e acredita-se que sempre 
comece com um comportamento alimentar inadequado (Lauder, 1999).

0 processo alimentar inadequado consiste em restrição de alimentos, rígidos esquemas alimentares, uso de pílulas e laxantes, vômitos e jejuns prolongados, e acredita-se serem esses comportamentos incentivados por uma imagem corpórea específica que os treinadores, as colegas de treino, a mídia e as próprias atletas idolatram (American ..., 1989).

O período da adolescência é muito importante do ponto de vista nutricional, pois nessa fase há grande desenvolvimento e crescimento dos indivíduos. Há maior demanda de substâncias nutritivas, interferindo na ingestão alimentar e aumentando as necessidades de nutrientes específicos. A inadequação da dieta pode retardar o crescimento e a maturação sexual (Dunker, 1999).

O adolescente, nesse período, está desenvolvendo e definindo sua identidade, auto-imagem e estilo de vida, e está se reajustando à vida social, familiar e escolar, de forma a tornar-se um ser adulto social e aceito pela sociedade. Esse reajuste é caracterizado por comportamentos de contestação da autoridade e quebra de padrões. 0 adolescente torna-se vulnerável, volúvel, seguidor de líderes, grupos e modismos, desenvolvendo preocupações ligadas ao corpo e à aparência (M antoanelli et al., 1997).

As atletas estão mais suscetíveis às opiniões da sociedade e pressões em relação ao desempenho, tornando-se altamente influenciáveis e com grandes possibilidades de desenvolver desordens alimentares. Não se sabe ao certo a prevalência dessas desordens nas atletas norte-americanas, mas a literatura relata índices que podem chegar a $62 \%$ em esportes nos quais há necessidade de baixo peso corporal. Nos Estados Unidos 65\% das adolescentes apresentam distúrbios alimentares (West, 1998; Lauder, 1999).

As atletas com desordens alimentares podem ter disfunção no ciclo menstrual, como a oligomenorréia ou a amenorréia. A prevalência, nos Estados Unidos, de irregularidades menstruais entre as esportistas, atinge índices de até $66 \%$ comparativamente ao restante da população feminina, o qual apresenta cifras de $2 \%$ a $5 \%$, mostrando que a ocorrência da amenorréia é maior em mulheres atletas em relação às não-atletas. A tualmente, a incidência de amenorréia e irregularidades menstruais vem aumentando em mulheres atletas e dançarinas (Calberg et al., 1983a; American..., 1989; Lauder, 1999).

Existe uma relação entre os distúrbios menstruais e a diminuição da massa óssea, decorrente da diminuição do estrógeno nas atletas com amenorréia (Lauder, 1999). As esportistas que não menstruam regularmente possuem risco maior de desenvolver osteoporose precoce em comparação àquelas com ciclos regulares e às não-atletas, e essa diminuição da massa óssea pode estar associada à maior susceptibilidade a fraturas em extremidades (Benson et al., 1995).

No passado, para explicar a amenorréia em atletas, enfocavam-se a perda de massa gorda ou peso, a redução energética e 0 excesso de exercício. Porém, não há evidências associando esses fatores. Os resultados contraditórios são explicados pelas grandes diferenças dos métodos e técnicas aplicados, assim como pelas diferenças do conceito de irregularidades menstruais de autor para autor, influenciando a análise estatística.

Amenorréia e osteopenia decorrentes do esporte podem ser definidas como uma condição clínica, não apenas pelo fato de serem mulheres atletas, mas como conseqüência de sua atividade física, devendo ser tratada e podendo influenciar o seu futuro (Burke, 1994).

A abordagem clínica de atletas adultas com amenorréia inclui exames físicos e endocrinológicos e suplementação de estrógeno e cálcio. Algumas dessas abordagens são provavelmente apropriadas para as adolescentes. Entretanto, a terapêutica hormonal não deve ser rotina nesses casos e estas devem ser encorajadas a diminuir a intensidade de atividade e aumentar o consumo alimentar (American..., 1989). 
Em vista do exposto, a partir de revisão bibliográfica da literatura, pretende-se, neste trabalho, discorrer sobre a amenorréia em adolescentes esportistas e relacioná-la com a osteoporose, a fim de auxiliar o profissional da área de nutrição na compreensão das variáveis que interferem na saúde destas atletas.

\section{A M E N ORRÉ IA E SUAS POSSÍVEIS CAUSAS}

O ciclo menstrual é caracterizado pelo intervalo entre um fluxo menstrual e o início do fluxo subseqüente. Na adolescência geralmente é de 21 a 40 dias e a duração do sangramento pode ser de dois a oito dias. A menarca (primeira menstruação) ocorre com o amadurecimento do eixo hipotálamo-hipófise-ovários (HHO), normalmente quando as adolescentes estão, segundo a classificação de Tanner dos caracteres sexuais secundários, em M3 e M4 de desenvolvimento mamário. Nos Estados Unidosa média de idade em que ocorre a menarca é 12,8 anos e no Brasil é 12,2 anos. Após a menarca, durante os dois primeiros anos, é comum existir irregularidade no ciclo menstrual, em virtude de o eixo $\mathrm{HHO}$ ainda estar amadurecendo, podendo durar de um a cinco anos e, no quinto ano, persistir em $20 \%$ das adolescentes. Geralmente esses ciclos são anovulatórios e, com a maturação progressiva do eixo, eles vão se tornando predominantemente ovulatórios (Beznos, 1993).

A amenorréia pode ser causada por várias razões, sendo a mais comum a fisiológica, decorrente da gravidez. As causas patológicas podem incluir anormalidades anatômicas, falha das gônadas e desordens do eixo HHO. Contudo, ela também pode ser desencadeada por estresse físico e/ou psicológico (Timmerman, 1996). Pesquisa nos Estados Unidos relacionou o atraso da menarca em adolescentes à pratica de atividade física, e para cada esporte detectou-se um atraso específico, como, por exemplo, no vôlei, no qual a média da menarca foi de 14 anos (Hale, 1984).
A amenorréia causada pelo esporte (AME) é definida como uma disfunção menstrual que ocorre em atletas. Engloba a amenorréia primária, a amenorréia secundária, ou mesmo mudanças no ciclo menstrual (Burke, 1994). De acordo com alguns, o atraso da menarca se relaciona com atividade física intensa antes da puberdade; conseqüentemente, há redução no acúmulo de massa óssea, resultando em diminuição do crescimento potencial (Broso \& Subrizi, 1996; Hirschberg \& Hagenfeldt, 1998). Entretanto, o seu mecanismo não é bem compreendido.

A AME está relacionada a um conjunto de fatores (excesso de treino, estresse fisiológico e psicológico, composição corporal) que, associados à dieta inadequada, resulta na interrupção dos ciclos menstruais normais. Esta disfunção deve ser vista como sintoma e não como diagnóstico, conforme aconselham Benson et al. (1995); Timmerman (1996); M cGee (1997).

Assim, ao se verificar o estresse ao qual atletas estão submetidas, devido às competições e compromissos associados a elas, pode-se concluir ser este um fator primordial para 0 desencadeamento do sintoma (Sanborn et al., 2000). Porém, segundo estudo submetendo corredoras a quatro testes psicológicos, a escala de depressão, ansiedade, obsessão e hipocondria entre as corredoras com e sem amenorréia era a mesma. A pesar desse resultado, as atletas referiam apresentar distúrbios menstruais causados pelo estresse, concluindo-se que o estresse psicológico pode afetar mais algumas atletas que outras, possivelmente em virtude de características individuais (Calberg et al., 1983a). Por outro lado, sabe-se que o estresse psicológico pode causar amenorréia mesmo em mulheres não praticantes de atividade física.

\section{Treino}

Os programas de treinamento de atletas não são uniformes, variando desde a quantidade ou duração das sessões, intensidade ou nível ou rendimento, até a freqüência das sessões (Burke, 
1994). A penas o exercício ou sua associação à perda de peso corpóreo podem contribuir para o desenvolvimento de distúrbios menstruais.

Há duas décadas já se verificava a possibilidade de o exercício intenso induzir irregularidades menstruais e inferia-se que $25 \%$ a $40 \%$ das atletas praticantes de esporte de longa duração e predominantemente aeróbio apresentavam menos de três ciclos por ano (Benson et al., 1995). Segundo referem Calberg et al. (1983a) esses distúrbios menstruais eram mais comuns em atletas campeãs e naquelas submetidas a treinamento físico mais exigente.

Em estudo envolvendo 32 bailarinas e comparando-as a controles (mulheres não praticantes de exercício e sem histórico de problemas menstruais), de idades semelhantes, as bailarinas apresentaram menos ciclos por ano. Além disso, de um total de oito mulheres, seis (75\% ) possuíam algum tipo de irregularidade menstrual associada ao aumento do treinamento durante as temporadas de balé; fora das temporadas, de um grupo de nove bailarinas, sete (78\% ) referiram maior regularidade menstrual (Cohen et al., 1982). Freqüência maior de amenorréia ou ciclos irregulares em bailarinas também foi descrito por Calabrese et al. (1983).

A explicação para esse fato está nas alterações hormonais ocorridas, as quais podem afetar o sistema reprodutivo. Atletas com amenorréia possuem quantidade de cortisol elevada em $25 \%$, enquanto as mulheres sedentárias ou as ciclistas apresentam pequena mudança em um período de 24 horas (Benson et al., 1995).

Conforme relatos de pesquisas, as atletas com irregularidades menstruais percorrem uma distância maior do que aquelas sem problemas menstruais (Burke, 1994). Por outro lado, estudo realizado durante seis meses, com estudantes universitárias com a função menstrual bem estabelecida, submetendo sua população a um treinamento específico de 3,5 horas por dia e ainda a um programa de corrida, no qual se foi progressivamente aumentando a distância, concluiu que a relação da mudança no ciclo menstrual com o aumento de treinamento só ocorreu quando houve perda de peso corporal (Bullen et al., 1984).

A relação entre amenorréia e quantidade de exercício é freqüentemente citada pela literatura na população de corredoras e bailarinas, mas não em grupos de ciclistas e nadadoras, mostrando maior prevalência no primeiro grupo (Cohen et al., 1982; Burke, 1994). Assim, pode-se inferir que o tipo de treino realizado e a diferença da composição corporal podem influenciar no surgimento do sintoma.

Corredoras e dançarinas sofrem grande elevação na temperatura central, podendo acarretar o interrompimento da regulação do sistema reprodutivo no hipotálamo; porém, essa elevação não ocorre em nadadoras e ciclistas. 0 possível controle de temperatura central entre estas esportistas talvez seja devido à sua constante exposição à água e ao vento, mas não há evidências fisiológicas para embasar essa teoria (Hale, 1984; Burke, 1994).

0 efeito do excesso de treino pode ser maior em jovens atletas. Frisch et al. (1981) estudaram a menarca e 0 ciclo menstrual de 21 nadadoras e 17 corredoras, e segundo observaram, $61,0 \%$ das atletas que começaram a treinar antes da menarca possuíam irregularidades menstruais e 22,4\% delas tinham quadro de amenorréia. Da população total apenas $16,7 \%$ apresentava ciclos regulares. Por meio deste estudo foi possível associar a amenorréia à idade das atletas, sendo mais comum naquelas com menos de 30 anos. Porém, outra pesquisa mostra idades semelhantes em atletas com e sem AME (Benson et al., 1995), evidenciando a contradição de resultados e impossibilitando conclusões.

Quanto ao histórico menstrual das esportistas, também existe controvérsia. Calberg et al. (1983a) referem que a maioria das atletas estudadas, antes de começar a treinar, já possuía irregularidades menstruais ou possuía ciclos regulares até $o$ início dos treinos. 
Em geral, as pesquisas mostram resultados divergentes na relação entre irregularidades menstruais e o volume de treino nos esportes. Estudos criteriosos quanto ao tipo de exercício, ao volume de treino, à intensidade, à freqüência ou a mudanças nesses fatores são necessários para se chegar a um consenso dos efeitos do treino sobre os ciclos menstruais (Burke, 1994).

\section{Peso}

A relação entre perda de peso e a disfunção menstrual não é bem compreendida, porém corredoras com amenorréia descreveram uma perda maior de peso em relação às não amenorréicas. A perda de peso pode ser 0 fator causador da AME, mas mesmo mulheres sedentárias também podem apresentar os sintomas (Benson et al., 1995).

Cohen et al. (1982), ao estudarem dançarinas com amenorréia, verificaram que estas possuíam um percentual menor de peso ideal em relação àquelas sem amenorréia. Frisch et al. (1980) notaram a mesma relação quando estudaram 89 dançarinas, das quais $15 \%$ apresentavam amenorréia secundária e 30\%, ciclos irregulares. 0 peso médio das dançarinas com amenorréia secundária foi de $44,9 \mathrm{~kg}$ e daquelas com ciclos irregulares foi de $45,9 \mathrm{~kg}$, contrastando com o peso de $47 \mathrm{~kg}$ nas dançarinas com ciclos normais.

Há relatos de mulheres com perda de peso associada a amenorréia apresentando anormalidades na secreção de gonadotropina e estrógeno e no metabolismo periférico de esteróides. A secreção de hormônio luteinizante nas atletas com AME é típica, mas, comparada àquelas sem $A M E$, essa secreção se encontra em quantidades anormais. Também foi descrito aumento de prolactina nas corredoras, o que poderia acarretar a parada do ciclo menstrual (Hale, 1984; Benson et al., 1995).

Apesar dos vários estudos mostrando a relação da perda de peso e a $A M E$, os fatos apontados na literatura são controversos, não se sabendo ao certo o que acarreta esta relação, não havendo assim conclusão definitiva.

\section{Gordura corporal}

A quantidade inadequada de gordura corporal pode ser a causa da disfunção menstrual e portanto, a composição corporal inadequada está relacionada à AME (Burke, 1994). A gordura periférica possui papel importante na conversão dos hormônios andrógenos em estrógenos. Quando existe diminuição dessa gordura, a quantidade de estrógeno diminui e os andrógenos aumentam, causando a parada dos ciclos menstruais. Não se sabe ao certo o mínimo necessário de gordura corporal para impedir a amenorréia (Hale, 1984). Frisch \& M cArthur (1974) propuseram que era necessário um mínimo de $17 \%$ de gordura corporal para haver a menarca e $22 \%$ para manter o ciclo regular. Esse trabalho foi muito criticado do ponto de vista dos métodos utilizados para estimar a gordura corporal e das análises estatísticas dos resultados (Burke, 1994). Estudo com técnicas mais apuradas, como a pesagem hidrostática, mostrou não haver diferenças significativas na porcentagem de gordura corporal entre atletas com AME e com ciclos regulares (Benson et al., 1995).

Em pesquisa realizada com 42 atletas praticantes de diferentes modalidades esportivas, Carlberg et al. (1983b) verificaram que aquelas com amenorréia possuíam quantidade inferior de gordura corporal em relação às não amenorréicas, sendo $13 \%$ e $16 \%$, respectivamente. As esportistas com amenorréia possuíam o peso corporal total muito inferior em relação àquelas sem os sintomas.

Diferentes porcentagens de gordura corporal entre atletas amenorréicas e eumenorréicas são constantemente descritas, e as divergências são freqüentes. Há relatos de que a quantidade de gordura corporal em corredoras com e sem amenorréia, submetidas ao mesmo tipo de treinamento, são semelhantes (Benson 
et al., 1995). Por outro lado, estudo realizado com 44 bailarinas e 23 controles, com ciclos menstruais regulares, encontrou uma massa gorda de 7,8 $\pm 0,4 \mathrm{~kg}$ no primeiro grupo e de $16,8 \pm 1,6 \mathrm{~kg}$ no segundo grupo, para um nível de significância inferior a 0,01 (Young et al., 1994).

Bale et al. (1996), ao estudar corredoras e ginastas, descrevem que somente $20 \%$ daquelas e $40 \%$ destas haviam apresentado a menarca, em comparação com o grupo controle, no qual 95\% já tinha menstruado.

De acordo com pesquisa realizada no Japão com 64 bailarinas, 15,6\% delas eram oligomenorréicas e 18,7\%, amenorréicas. As bailarinas amenorréicas apresentavam um percentual de gordura significativamente inferior às eumenorréicas, $15,72 \%$ e 17,95\%, respectivamente (To et al., 1997).

\section{Perda de estoques específicos de gordura}

A perda de depósitos específicos de gordura pode ser a chave para determinar disfunção menstrual, pois a gordura corporal é um fator regulador da função endócrina. 0 metabolismo do tecido adiposo difere de região para região em mulheres durante a gravidez e a lactação. A atividade lipolítica na região do quadril, das pernas e das nádegas é maior do que a na região abdominal em mulheres grávidas e em lactação, e nessa área a ação da lipase lipoprotéica é maior, porém mais pronunciada durante a gravidez. A gordura depositada nessas regiões possui papel importante no processo da reprodução feminina. Supõe-se que, quando esses estoques estão abaixo de determinada quantidade, existe a impossibilidade de engravidar ou amamentar. A depleção desses estoques em esportistas pode ser uma possível explicação para as disfunções menstruais, sinalizando que o corpo não pode sustentar a fertilidade. Não se sabe exatamente as diferenças na distribuição de gordura entre atletas com e sem amenorréia, mas sua existência é conhecida. Especula-se também se o corpo tentaria manter essa gordura, principalmente a relacionada à reprodução, modificando o metabolismo basal ou a eficiência do aproveitamento dos nutrientes ingeridos (Burke, 1994).

\section{Inadequação nutricional}

A ingestão energética insuficiente, mesmo sem atividade física, é um dos fatores que contribuem para a disfunção menstrual. A relação entre o consumo nutricional e o treino permanece sem esclarecimento.

A redução de energia ingerida não está relacionada com o aparelho reprodutor feminino, porém a desnutrição aguda, provocada por essa restrição, está associada ao surgimento da amenorréia. Quando o organismo privado tem acesso novamente ao alimento, essa situação é revertida. Esse fato foi demonstrado na Segunda Guerra M undial, pois, em conseqüência da falta de alimento, muitas mulheres tornaram-se amenorréicas. Com o final da guerra, o acesso à alimentação foi restabelecido, assim como os ciclos menstruais (Beznos, 1993).

As dançarinas e corredoras, as quais apresentam maior incidência de amenorréia, geralmente são submetidas a dietas nutricionalmente pobres, consumindo menos macro e micronutrientes do que necessitam (Burke, 1994).

A quantidade energética ingerida não guarda relação com as irregularidades menstruais, porque os grupos de atletas com e sem AME tiveram uma ingestão energética semelhante (Benson et al., 1995; Fruth \& Worrel, 1995). Esses estudos avaliaram apenas a quantidade energética como fator causador das disfunções menstruais, mas outros nutrientes da dieta também podem ter influência. Ao se comparar a ingestão alimentar de atletas com e sem AME, Drinkwater et al. (1984) notaram que o primeiro grupo consumia menos energia e menos lipídios 
(1622 kcal e 56,7 g, respectivamente), em relação ao segundo grupo (1965 kcal e 79,3 g).

Kaiserauer et al. (1991), ao compararem corredoras com amenorréia, corredoras com ciclos regulares e o grupo controle composto de mulheres sedentárias com ciclos regulares, com relação ao estrógeno, características físicas e ingestão de nutrientes, observaram que as atletas com AME consumiam menos lipídios, carnes vermelhas e energia, quando comparadas àquelas com ciclos regulares. Não houve diferença entre gordura corporal, peso ou nível de exercício entre os dois grupos de corredoras.

O exercício em atletas com AME não acarreta necessariamente aumento da taxa de metabolismo basal (TM B). Na maioria dos casos a diminuição da TMB pode ser atribuída unicamente às perdas de massa magra (Burke, 1994).

De acordo com estudos de Hill et al. (1987), depois de oito semanas de dieta com $500 \mathrm{kcal}, 0$ declínio total da TM B no grupo que fazia atividade física foi de $19,1 \%$ e no grupo sem atividade foi de $17,3 \%$. Fato similar foi observado por Phinney et al. (1988). A pesar desses resultados, nem toda mulher atleta possui alteração na TMB.

Segundo relataram Deuster et al. (1986), comparando peso, gordura corporal e parâmetros dietéticos em corredoras, as atletas com AM E não apresentavam diferenças significativas de peso, gordura corporal e carga de exercício em relação àquelas sem os sintomas, porém a quantidade de lipídios ingerida era significativamente menor e a quantidade de fibras era alta.

Lloyd et al. (1987) encontraram uma relação positiva entre o consumo de fibras e as irregularidades menstruais. 0 aumento deste consumo também está relacionado com alterações no metabolismo do estrógeno. Contudo, há evidências de que a ingestão de fibras entre as atletas com ou sem o sintoma são semelhantes (Fruth \& Worrell, 1995).

O baixo consumo de proteínas pode contribuir para o surgimento da amenorréia. No entanto, atletas com baixo consumo de energia têm ingestão protéica inferior à daquelas com consumo energético normal. Assim, estudos mostraram que a ingestão protéica em si não possui relação com as irregularidades menstruais (Hale, 1984; Lloyd et al., 1987; Fruth \& Worrell, 1995).

Em virtude do conjunto de relações descritas, o grupo de atletas vegetarianas possui risco maior de ter $\mathrm{AME}$ em comparação com 0 grupo de não vegetarianas, pois o padrão dietético daquele grupo é de consumo baixo em lipídios e carnes, e alto em fibras (Burke, 1994). Ao se analisar o padrão dietético de 11 atletas com AME e 15 com ciclos regulares, verificou-se entre as corredoras com ciclos menstruais regulares ingestão de carne cinco vezes maior do que entre aquelas com AME. Para essa pesquisa foram classificadas como vegetarianas as atletas com consumo inferior a 200 gramas de carne por semana. Assim, das corredoras com AME, 82\% eram vegetarianas, e das com ciclos regulares, $13 \%$ seguiam esse mesmo padrão alimentar. As vegetarianas com AME consumiam cerca de $68 \mathrm{~g}$ de lipídios por dia, enquanto o outro grupo de atletas consumia cerca de $98 \mathrm{~g}$ por dia (Benson et al., 1995). Kaiserauer et al. (1991) também encontraram essa relação e descreveram que $25 \%$ das atletas com amenorréia eram vegetarianas.

Apesar de os estudos apresentarem divergências entre a relação da dieta e as irregularidades menstruais, sabe-se que 0 hipotálamo detecta os balanços nutricionais. Caso exista algo fora do padrão, um sistema de proteção é ativado, atuando geralmente através da supressão do sistema reprodutivo e resultando na disfunção menstrual (Fruth \& Worrell, 1995).

Bailarinas, corredoras e ginastas apresentam freqüentemente amenorréia e oligomenorréia do que outros grupos de esportistas como nadadoras e ciclistas. Corredoras de alto nível competitivo podem apresentar amenorréia, mas parece que existem outros fatores influenciando o seu surgimento, pois a tolerância individual é muito variável. Em outras adolescentes o que pode estar envolvido é o estresse, 
treinamento intensivo, ou o consumo de dieta inadequada.

\section{O STEOPOROSE} E A MENORRÉIA

A osteoporose é uma doença em que ocorre importante perda de densidade óssea, aumentando assim sua fragilidade e a tendência às fraturas. Nos Estados Unidos essa doença atinge cerca de 25 milhões de pessoas, ocasiona 1,5 milhões de fraturas por ano e representa cerca de 6 bilhões de dólares gastos por ano, tornando-a sério problema de saúde pública (West, 1998). As fraturas causadas por estresse são comuns entre as atletas e ocorrem principalmente nos ossos do sacro, na pélvis e na cabeça do fêmur (Zeni et al., 2000).

As bailarinas e as corredoras são as mais afetadas pelas fraturas decorrentes de estresse. Nas bailarinas a fratura mais comum é na base do segundo metatarso, e, segundo descreve a literatura, o risco para sua ocorrência está ligado a treino intenso, atraso na menarca e alimentação inadequada (O'M alley et al., 1996; Bennel \& Brukner, 1997).

É importante considerar que o osso é um tecido vivo o qual se encontra em constantes ciclos de mineralização e reabsorção e qualquer mudança nesse balanço pode causar perdas ou ganhos de massa óssea.

0 esqueleto humano é formado por dois tipos de ossos, um com componentes densos (corticais) e outro com esponjosos (trabeculares), geralmente mais vulneráveis à doença (Beck \& Schoemaker, 2000). Cada tipo de osso tem sua formação máxima em diferentes estágios da vida. A aquisição de massa óssea é gradual durante a infância e acelerada durante a adolescência, até o indivíduo atingir a maturidade sexual. Quase $50 \%$ da massa óssea é adquirida nessa fase, pois o acúmulo de cálcio é triplicado. Contudo, a idade em que o pico de formação óssea (FO) ocorre permanece controverso na literatura (Burke, 1994; Nattiv \& Armsey, 1997). Esse pico ocorre geralmente entre 18 e 25 anos de idade, sendo no sexo feminino por volta dos 20 anos e talvez 16 anos nas mulheres com puberdade precoce (Wiggins \& Wiggins, 1997). Em contrapartida, Nattiv \& Armsey (1997) e West (1998) afirmam que até os 18 anos $90 \%$ da massa óssea já se formou, pois o ápice ocorre durante o estirão da adolescência. Por causa de uma série de fatores, alguns deles nutricionais e outros hormonais (Krebs, 2001), o pico de desenvolvimento ósseo pode ser baixo, acarretando risco futuro de fraturas, e maximizá-lo é uma forma de prevenção contra a osteoporose e a osteopenia.

Após essa formação começa a depleção, ou seja, a perda de densidade óssea, a qual pode chegar em torno de 0,3\% a 0,5\% por ano. Durante a menopausa as perdas ósseas podem aumentar dez vezes mais, chegando a 3\% por ano, sendo essa perda um processo natural da idade. A osteoporose não é uma doença restrita aos idosos e é comumente vista em mulheres jovens com amenorréia.

Alguns fatores que predispõem ao desenvolvimento da osteoporose são o sedentarismo, o tabagismo, a falta de estrógeno e a ingestão alimentar e o consumo de cálcio inadequados (West, 1998; Patrick, 1999). Assim, a atividade física e a dieta rica em cálcio podem ser associados positivamente ao aumento da massa óssea em crianças e adolescentes.

A redução de massa óssea está intimamente ligada à tendência de sedentarismo ao longo dos anos, pois há acentuada diminuição na estimulação mecânica dos osteoblastos (Beck $\&$ Schoemaker, 2000), porém o efeito protetor do exercício sobre o esqueleto é perdido naquelas atletas com menarca atrasada ou amenorréia. Um estud o de caso controle realizado por Rencken et al. (1996), envolvendo 49 atletas, verificou que a idade da menarca, assim como os períodos prolongados de amenorréia, está relacionada com a densidade óssea. Portanto, 0 atraso na menarca e a amenorréia se associam à diminuição na massa óssea. 
Jovens atletas com AME têm perda óssea de $2 \%$ a $6 \%$ por ano, e por volta dos 20 anos sua massa óssea é equiparável a uma mulher de 60 anos de idade. Essas perdas podem ser irreversíveis, pois o pico de FO nunca foi atingido, e então a massa óssea perdida nunca será completamente recuperada, mesmo a mulher passando a ter ciclos menstruais normais, com reposição de estrógeno e suplementação de cálcio. Possivelmente uma atleta com amenorréia demoraria de 10 a 15 anos para ter sua massa óssea restaurada (West, 1998; Lauder, 1999).

Não se tem conhecimento ao certo da prevalência de baixa quantidade de massa óssea em atletas, mas sabe-se que naquelas com $A M E$ essa quantidade é inferior em relação àquelas com ciclos menstruais regulares (Fruth \& Worrell, 1995).

0 efeito do estrógeno na massa óssea não está bem esclarecido, porém foi descrito ser a exposição ao hormônio inversamente proporcional ao risco de desenvolver osteoporose.

Assim, as mulheres que menstruaram mais cedo foram expostas mais cedo ao hormônio e atingiram o pico de $\mathrm{FO}$, ao contrário daquelas com atraso na menarca ou com amenorréia, as quais foram expostas por menos tempo ao estrógeno, diminuindo a massa óssea e aumentando o risco de desenvolver a doença (Fruth \& Worrell, 1995). Conforme relataram Nguyen et al. (1995), a duração de exposição ao estrógeno, somando os anos de ciclo menstrual regular e a terapia hormonal pós-menopausa, se associava a aumento da massa óssea e a redução de fraturas. Portanto, além de o estrógeno auxiliar no aumento da massa óssea, também reduz o risco de fraturas causadas pela osteoporose (Ericson \& Sevier, 1997).

Ao estudar corredoras entre 16 e 30 anos, Pettersson et al. (1999) concluíram que as atletas amenorréicas e com deficiência de estrógeno possuíam menor quantidade de massa óssea em relação às demais.

O emprego de terapia de reposição hormonal em mulheres pós-menopausa mostrou ser possível um aumento na densidade óssea de
$1,7 \%$ nos quadris e de 3,5\% a 5,0\% na espinha dorsal. Por outro lado, as mulheres não submetidas a esta terapia apresentaram perda de massa óssea de $1,7 \%$ nos quadris e $1,8 \%$ na espinha dorsal (Ericson \& Sevier, 1997).

Outros trabalhos também relacionaram a manutenção da densidade do osso à progesterona, pois esta contribui para a formação óssea e acelera a sua remodelação, enquanto o estrógeno previne a reabsorção do osso. Com a queda na produção da progesterona, na fase lútea, há diminuição na densidade óssea, mesmo com níveis de estrógeno e ciclos menstruais normais (Burke, 1994; Fruth \& Worrell, 1995).

O consumo do cálcio está diretamente relacionado à massa óssea. Em gemelares, ao se suplementar esse micronutriente em um dos irmãos, verificou-se aumento significativo de sua massa óssea em relação àquele que permaneceu ingerindo quantidades de acordo com as Recommended Dietary Allowances (RDA) (National Research..., 1989).

Especula-se se as atletas consomem cálcio em quantidade inferior à que o National Research Council de 1989 preconizava, 1200 mg ao dia; com o aumento dessa recomendação, de acordo com as Dietary Reference Intakes (DRI) (Institute...,1998), para $1300 \mathrm{mg}$ ao dia, essa inadequação será ainda maior. O consumo inadequado, associado às especificidades nutricionais da adolescência, fase na qual existe necessidade de maior ingestão de nutrientes, em virtude do crescimento e desenvolvimento, pode influenciar o surgimento de osteoporose precoce (National Research..., 1989; Burke, 1994; Fruth \& Worrell, 1995; Dunker, 1999). Contudo, segundo Pettersson et al. (1999) verificaram, o consumo de cálcio nas corredoras amenorréicas e eumenorréicas era semelhante ou acima do recomendado pelo National Research... (1989). Portanto, a saúde óssea e o consumo de cálcio durante a puberdade possuem relação direta.

Por outro lado, o balanço do cálcio no organismo não está somente relacionado à sua ingestão. Outrosfatores da dieta podem influenciar 
negativamente, como altas quantidades de sal, proteínas, fósforo, cafeína e álcool (National Research..., 1989).

0 método mais efetivo contra a osteoporose é a prevenção; portanto, durante o crescimento, para maximizar o desenvolvimento ósseo, deve-se priorizar a ingestão adequada de cálcio e a prática de atividade física apropriada (Beck \& Schoemaker, 2000). 0 consumo alimentar é fator decisivo para a prevenção da amenorréia $\mathrm{e}$, conseqüentemente, da osteoporose, tornando imprescindível o seu conhecimento na população de adolescentes atletas, pois através dele podemse tomar medidas preventivas, como 0 aumento da ingestão alimentar, a suplementação do cálcio e a diminuição da atividade física.

\section{O N C L U S Ã O}

A fase de desenvolvimento e crescimento de um adolescente é período crítico e de fundamental importância na qualidade de vida futura do indivíduo. Os profissionais da área de saúde devem dispor das ferramentas necessárias para esse desenvolvimento e crescimento ocorrerem de maneira saudável. No caso das adolescentes atletas, elas têm as necessidades específicas da idade e as decorrentes da atividade física profissional, e a somatória dessas duas características as tornam um grupo muito especial.

A osteoporose é uma doença que se tornou um problema de saúde pública ao longo dos anos. 0 processo de perda óssea é algo natural com a idade, mas o grupo feminino de adolescentes atletas possui risco de desenvolvê-la precocemente, pois a incidência de amenorréia vem aumentando dentro dessa população, e essa disfunção no ciclo menstrual se relaciona com o desenvolvimento da osteoporose.

M uitas especulações foram feitas para se chegar às causas da amenorréia nas atletas, porém as conclusões permanecem contraditórias. Quanto mais precoce for o diagnóstico provavelmente menores serão as chances de prejuízo ósseo.
A pesar de as causas serem desconhecidas existem tratamentos que devem ser utilizados logo após o diagnóstico ser determinado.

Um plano de educação nutricional deve ser utilizado para se conscientizar as atletas e as pessoas envolvidas sobre a importância da dieta equilibrada e adequada, a qual pode impedir o desenvolvimento da amenorréia e, conseqüentemente, prevenir a osteoporose precoce. Além disso, garantir a ingestão de cálcio em quantidade de acordo com o preconizado pelas DRI também é fator de proteção contra a doença.

M uitos fatores interferem na interpretação do que a literatura nos oferece: desde o que diz respeito à escolha do método estatístico, à qualidade dos estudos, ao tamanho do estudo, até a sua localização geográfica.

Estatísticas adequadas contribuem para a monitorização da saúde das populações, auxiliando na identificação de grupos de risco e implantação e priorização de ações.

Maiores estudos são necessários para a elucidação das causas da amenorréia e de suas conseqüências, assim como da sua relação com o desenvolvimento da osteoporose precoce. No entanto, estão evidentes os efeitos metabólicos ou endocrinológicos em indivíduos com gordura corporal inferior ao necessário para a sua manutenção, deprimindo o metabolismo ou reduzindo a produção de estrógeno.

\section{REFERÊ NCIAS B I B L I O G RÁ F I C A S}

AMERICAN ACADEMY OF PEDIATRICS. Amenorrhea in adolescent athletes. Pediatrics. 1989; 84(2). [online]. Available from: <URL: http://www.aap.org/ policy/02626.html>. [2000 Nov 3].

BALE, P., DOUST, J., DAWSON, D. Gymnasts, distance runners, anorexics body composition and menstrual status. Journal of Sports Medicine Physical Fitness, v.1, n.36, p.49-53, 1996.

BECK, B.B., SCHOEM AKER, M.R. Understanding key risk factors and therapeutic options. The Physician 
and Sports Medicine. 2000; 28 (2). [online]. Available from: <URL: http://www.physician and sports medicine.com/issues/2000/02_00/beck. htm>. [ 2001 agosto 28].

BENNEL, K.L., BRUKNER, P.D. Epidemiology and site specificity of stress fractures. Clinical Sports Medicine, v.16, n.2, p.179-196, 1997.

BENSON, J.E., EISENM AN, P.A., HEINRICH, K.K. The relation of caloric and fiber and metabolic rate to athletic amenorrhea In: KIES, C.V., DRISKELL, J.A. Sports nutrition: mineral and electrolytes. New York: CRC Press; 1995. p.305-315.

BEZNOS, G.W. Distúrbios menstruais. In: COATES, V., FRANÇOSO, L.A., BEZNOS,G.W. Medicina do adolescente. São Paulo : Sarvier, 1993. p.207-210.

BROSO, R., SUBRIZI, R. Gynecologic problems in female atheles. M inerva Gynecology, v.48, n.3, p.99-106, 1996.

BULLEN, B.A., SKRINAR, G.S., M ERING, B.A., TURNBULL, B.A., M CARTHUR, A.W. Induction of menstrual disorders by strenuous exercise in untrained women. New England Journal Medicine, v.312, p.1349-1353, 1984.

BURKE, L. Sports amenorrhea, osteopenia, stress fratures and calcium. In: BURKE, L. DEAKIN, V. Clinical sports nutrition. Sidney : M CGraw-Hill, 1994. p.200-226.

CALABRESE, L.H., KIRKENDALL, D.T., FLOYD, M. Menstrual abnormalities, nutritional patterns and body composition in female classical ballet dancers. Physician Sports M edicine, v.11, p.86-90, 1983.

CALLAHAN, L.R. The evolution of the female athlete: progress and problems. Pediatrics Annals, v.29, n.3, p.149-153, 2000.

CARLBERG, K.A., BUCKM AN, M.T., PEAKE G.T. Exercise and the menstrual cycle. In: APPENZELLER, O., ATKINSON, R. Sports medicine: fitness, training, injuries. 2.ed. Baltimore : Urban \& Schwarzenberg, 1983a. p.131-143.

CARLBERG, K.A., BUCKMAN, M.T., PEAKE, G.T., RIEDESEL, M.L., Body composition of oligo/ amenorrheic athetes. Medicine Science Sports Exercise, v.15, n.3, p.215-217, 1983b.
COHEN, J.L., CHUNG, S.K., MAY, P.B. Exercise, body weight, and amenorrhea in professional ballet dancers. Physician Sports M edicine, v.10, p.92-100, 1982.

DEUSTER, P.A., KYLE, S.B., M OSER, P.B., VIGERSKY, R.A., SINGH, A.,SCHOOMAKER, E.B. Nutritional intakes and status of highly trained amenorrheic and eumenorrheic women runners. Fertil Steril, v.46, n.4, p.636-643, 1986.

DRINKWATER, B.L., NILSON, K., CHESTNUT, C.H., BREM NER, W.J. SHAINHOLTZ, S., SOUTHWORTH, $M . B$. Bone mineral content of amenorrheic and eumenorrheic athletes. New England Journal of Medicine, v.311, n.5, p.277-281, 1984.

DUNKER, K.L.L. Avaliação nutricional e comportamento alimentar de adolescentes com sintomas de anorexia nervosa. São Paulo, 1999. Dissertação (Mestrado) - Faculdade de Saúde Pública, Universidade de São Paulo, 1999.

ERICSON, S.M., SEVIER, T.L. Osteoporosis in active women: prevention, diagnosis, and treatment. The Physician and Sports Medicine. 1997; 25: 11. [online]. Available from: <URL: http://www.physician and sports medicine.com/issues/1997/11nov/ erickson.htm>. [2001 agosto 28].

FRISCH, R.E., MCARTHUR, J.W. Menstrual cycles: fatness as a determinant of minimum weight for height necessary for their maintenance or onset. Science, v.185, n.4155, p.949-951, 1974.

FRISCH, R.E., Wyshak, G., VINCENT, L. Delayed menarche and amenorrhea in ballet dancers. New England Journal of Medicine, v.303, n.1, p.17-19, 1980.

FRISCH, R.E., GOTZ-WELBERGEN, A.V., M CARTHUR, J.W., ALBRIGHT, T., WITSCHI, J., BULLEN, B., BIRNHOLZ, J., REED, R.B., HERM ANN, H. Delayed menarche and amenorrhea of college athletes in relation to age on onset training. Journal of American Medicine Association, Chicago, v.246, n.4, p.1559-1563, 1981.

FRUTH, S.J., WORRELL, T.W. Factors associated with menstrual irregularities and decreased bone mineral density in female athletes. Journal of Orthopedics Sports Physician Therapeutic, v.22, n.1, p.26-38, 1995. 
HALE, R.W. Factors important to women engaged in vigorous physical activity. In: STRAUSS, R.H. Sports medicine. Philadelphia : WB Sauderes, 1984. p.258-263.

HILL, J.O., SPARLING, P.B., SHIELDS, T.W., HELLER, P.A. Effects of exercise and food restriction on body composition and metabolic rate in obese women. American Journal of Clinical Nutrition, Bethesda, v.46, n.4, p.622-630, 1987.

HIRSCHBERG, A.L., HAGENFELDT, K. Athletic amenorrhea and its consequences. Hard physical training at an early age can cause serious bone damage. Lakartidningen, v.95, n.50, p.5765-5770, 1998.

INSTITUTE OF MEDICINE (US). Dietary references intakes for calcium, phosphorus, magnesium, vitamin $D$ and fluoride. Washington DC: National Academy Press, 1998. 432p.

KAISERAUER, S., SNYDER, A.C., SLEEPER, M., ZIERATH, J. Nutritional, physiological and menstrual status of distance runners. Medicine Science Sports Exercise, v.23, p.15, 1991.

KREBS, N.F. Bioavailability of dietary supplements and impact of physiological state: infants, children and adolescents. Journal of Nutrition, v.131, n.4, p.1351S-1354S, 2001. Supplement.

LAUDER, T.D. The female athlete triad: prevalence in military women. Military Medicine, v.164, n.9, p.630-635, 1999.

LLOYD, T., BUCHANAN, J.R., BITZER, S. Interrelationships of diet, athletic activity, menstrual status, and bone density in collegiate women. American Journal of Clinical Nutrition, Bethesda, v.46, n.4, p.681-684, 1987.

MANTOANELLI, G., BITTENCOURT, V.B., PENTEADO, R.S., PEREIRA, I.M.T.B., ALVAREZ, M.C.A. Educação nutricional: uma resposta ao problema da obesidade em adolescente. Revista Brasileira de Crescimento e Desenvolvimento Humano, v.2, n.7, p.85-93, 1997.

MCGEE, C. Secondary amenorrhea leading to osteosporosis incidence and prevention. Nurse Practitioner, v.22, n.5, p.38-65, 1997.

NGUYEN, T.V., JONES, G., SAM BROOK, P.N. Effects of estrogen exposure and reproductive factors on bone mineral density and osteoporotic fractures. Journal of Clinical Endocrinology M etabolism, v.80, n.9, p.2709-2714, 1995.

NATIONAL RESEARCH COUNCIL (USA). Recommended dietary allowances. Washington DC: National Academy Press, 1989. 283p.

O'M ALLEY, M.J., HAM ILTON, W.G., MUNYAK, J., DEFRANCO, M.J. Stress fractures at the base of the second metatarsal in ballet dancers. Foot Ankle International, v.17, n.2, p.89-94, 1996.

NATTIV, A., ARMSEY JR, T.D. Stress injury to bone in the female athlete. Clinics Sports Medicine, v.16, n.2, p.197-224, 1997.

PATRICK, L. Comparative absorption of calcium sources and calcium citrate malate for the prevention of osteoporosis. Alternative M edicine Review, v.4, n.2, p.74-85, 1999.

PETTERSSON, U., STALNACKE, B., ATHÉNIUS, G., HENRIKSSON-LARSÉN, K., LORENTZON, R. LOW bone mass density at multiple skeletal sites, including the appendicula skeleton in amenorrheic runners. Calcified Tissue International, v.64, n.2, p.117-125, 1999.

PHINNEY, S.D., LaGRANGE, B., O'CONNEL, M. DANFORTH, E. Effects of aerobic exercise on energy expenditure and nitrogen balance during very low dieting. M etabolism, v.37, n.8, p.758-765, 1988.

RENCKEN, M.L., CHESNUT, C.H., DRINKWATER, B.L. Bone density at multiple skeletal sites in amenorrheic athletes. JAM A, Chicago, v.276, n.3, p.238-240, 1996.

SANBORN, C.F., HOREA, M., SIEM ERS, B.J., DIERINGER, K.L. Disordered eating and female athlete triad. Clinical Sports Medicine, v.19, n.2, p.1999-1213, 2000.

TIM MERM AN, M.G. Medical problems of adolescent female athletes. W isconsin M edical Journal, v.95, n.6, p.351-354, 1996.

TO, W.W., WONG, M.W., CHAN, K.M. Association between body composition and menstrual dysfunction in collegiate dance students. Journal Obstetric Gynecology Research, v.23, n.6, p.529-535, 1997. 
WEST, R.V. The female athlete. The triad of disordered eating, amenohrrea and osteporosis. Sports Medicine, v.26, n.2, p.63-71, 1998.

WIGGINS, D.L., WIGGINS, M.E. The female athlete. Clinical Sports Medicine, v.16, n.4, p.593-612, 1997.

YOUNG, N., FORM ICA, C., SZM UKLER, G., SEEM AN, E. Bone density at weight-bearing and nonweight-bearing sites in ballet dancers: the effects of exercise, hypogonadism, and body weight.
Journal Clinical Endocrinology Metabolism, Duluth, v.78, n.2, p. 449-454, 1994.

ZENI, A.L., STREET, C.C., DEM PSEY, R.L., STATON, M. Stress injury to the bone among women athletes. Physician Medicine Rehabilitation Clinics North American, v.11, n.4, p.929-947, 2000.

Recebido para publicação em 29 de março e aceito em 5 de novembro de 2001 